\title{
Un informativo, dos enfoques: la actualidad política vasca en ETB durante los mandatos de lbarretxe y López
}

institucional.us.es/ambitos/

José María Pastor González

Universidad del País Vasco

josemari.pastor@ehu.es

\section{Resumen}

Este trabajo tiene como objeto de estudio la información sobre política vasca difundida por ETB en el informativo 'Gaur Egun' durante los mandatos de los lehendakaris Juan José Ibarretxe y Patxi López. El análisis y la comparación de los contenidos de los informativos de ambos períodos muestran dos modelos diferentes a la hora de abordar el tratamiento de la información política. En el primero de ellos se otorga mayor importancia a estas noticias. En el segundo, la información política vasca no ocupa un lugar tan relevante y se retringe más claramente al ámbito geográfico de la Comunidad Autónoma Vasca.

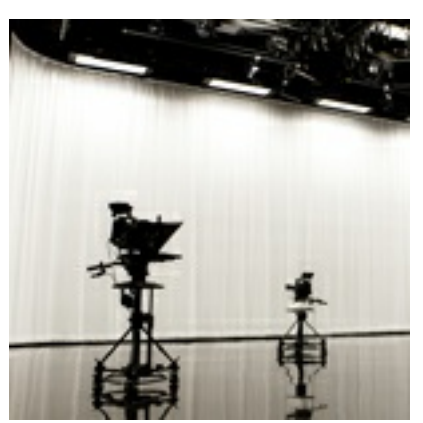

\section{Palabras clave}

ETB, información política vasca, Ibarretxe, Patxi López.

\begin{abstract}
This work aims to study the information about Basque politics broadcasted by ETB in the news program 'Gaur Egun' during the terms of Juan Jose Ibarretxe and Patxi Lopez, presidents of the Basque Autonomous Government. The analysis and comparison of the contents of the program in both periods show us two different models to address and process the political information. In the first of them Basque politics have a big importance. In the second one, that kind of news does not seem so relevant and is much more limited to the geographical area of the current Basque autonomous region.
\end{abstract}

\section{Keywords}

ETB, Baque political news, Ibarretxe, Patxi López.

\section{INTRODUCTION}

El presente trabajo aborda el tratamiento de la información política vasca en el ente público Euskal Telebista-Televisión Vasca durante dos períodos de tiempo diferentes, correspondientes a los mandatos de los lehendakaris Juan José Ibarretxe (PNV) y Patxi López (PSE-EE). En él se analiza cómo el cambio de gobierno influyó en el enfoque de la actualidad política vasca en los informativos de este medio de comunicación.

En el año 2009 el socialista Patxi López fue elegido lehendakari, interrumpiéndose así la hegemonía ejercida durante casi tres décadas por políticos nacionalistas como máximos dirigentes de la Comunidad Autónoma Vasca. Esta alternancia se produjo por primera vez en el gobierno vasco desde que el estatuto de autonomía de Gernika fuera aprobado y se reinstaurara esa institución autonómica. Este estudio toma como referencia los años 2008 y 2010 para analizar de qué manera afectó el cambio de gobierno en la producción de noticias y en los criterios a seguir por parte de ETB a la hora de tratar la información concerniente a la política vasca. Con ello se pretende comparar los principios y contenidos informativos vigentes durante esos dos mandatos a la hora de tratar temas o sucesos relacionados con ese ámbito susceptibles de ser difundidos por su importancia o repercusión en la opinión pública.

La televisión pública vasca comenzó su difusión en 1983. La primera emisión de ETB tuvo lugar en la 
Nochevieja del año 1982, con el discurso del entonces lehendakari Carlos Garaikoetxea. El inicio del año 1983 marcaba así un hito histórico, no tan solo en el mundo de la comunicación audiovisual en general y del ámbito vasco en particular, sino también en el todavía incipiente camino hacia la normalización de la lengua vasca. El nacimiento de la televisión pública en euskara satisfizo la demanda histórica de la población vasca: tener un medio de comunicación que le informara en su propia lengua acerca de la actualidad, tuviera esta su origen en el País Vasco o fuera de él. Por primera vez, los euskaldunes -personas de habla vasca- a ambos lados de los Pirineos disponían de un medio de comunicación audiovisual público en la lengua propia del país, según el estatuto de autonomía de Gernika, o en su lengua materna o natural en el caso de los vascoparlantes que vivían fuera de la Comunidad Autónoma Vasca. La televisión vasca tenía en consideración los siete territorios donde el euskara es hablado en la actualidad: al sur de los Pirineos las provincias de Álava, Bizkaia, Gipuzkoa y Navarra; al norte, las tierras labortanas, bajo-navarras y suletinas.

Si bien anteriormente se habían realizado emisiones en vasco -la primera de ellas desde la corresponsalía de la cadena pública francesa FR3 en Bayona en el año 1971; la segunda, desde el centro regional de TVE en Bilbao, en el año 1974- no fue hasta aquel año 1983 cuando una televisión pública -ETB, creada gracias a las competencias otorgadas al País Vasco en el estatuto de autonomía- comenzó a emitir tomando en consideración la información de todos los territorios de habla vasca con la pretensión de superar las dificultades reales, más legales que técnicas, para poder llegar a todos los hogares del País del Euskara, como etimológicamente se podría traducir el término Euskal Herria.

ETB no solo inserta el euskara en un nuevo mundo, en el campo audiovisual, como metáfora de la modernidad. La televisión vasca también sirve para estructurar los territorios vascoparlantes por encima de divisiones administrativas o fronteras estatales. En ese sentido, se puede decir que el ente público unifica esos territorios, y transciende la realidad y statu quo políticos vigentes para dotar de mayor poder simbólico al inconsciente colectivo euskaldun en torno al concepto de Euskal Herria. La televisión vasca no hace sino ayudar a construir la realidad social, tal y como han hecho hasta ahora todos los medios audiovisuales, según indica Joan Ferrés: "Con el advenimiento de la televisión se incrementó extraordinariamente el porcentaje de conocimientos humanos que son simples creencias. Es mínima la parte de conocimientos sobre el mundo que la persona puede verificar empíricamente. Nuestra imagen de la realidad es básicamente la que nos ofrecen los media, y sobre todo la televisión. De ahí la importancia de su control social" (Ferrés, 1994, 79).

Es en esa labor de crear la imagen de la realidad donde el control social y, por ende, el control político de la televisión cobra especial relevancia. La televisión vasca no constituye una excepción en este sentido. Desde su misma creación, incluso antes de su nacimiento oficial, el gobierno de la CAV, liderado por el Partido Nacionalista Vasco, buscó fuera de las fronteras del estado español el modelo y las referencias informativas que sirvieran como base para la creación de ETB. EI PNV quería de esta manera alejarse de las connotaciones "imperiales" que España suscitaba entre los medios nacionalistas vascos y gran parte de la opinión pública de la Comunidad Autónoma Vasca.

Así lo señaló Richard Maxwell en su libro The Spectacle of Democracy. Spanish Television, Nationalism and Political Transition, publicado en 1995, cuando ya habían pasado más de diez años desde que ETB iniciara sus emisiones: "Hace diez años, en el momento en que se creó ETB, muchos políticos vascos veían a España como un estado extranjero, como un poder imperial. En vez de dirigirse al estado, los vascos prefirieron mirar hacia otro sitio para pedir consejo y recoger experiencias, y así crear su nuevo sistema de televisión" (Maxwell, 1995, 140).

Por entonces el PSOE gobernaba en España. Según Maxwell, el hecho de que el gobierno vasco estuviera fuera del área de control de los socialistas posibilitó la creación de la televisión y radio públicas vascas:

"El hecho de que el gobierno vasco, controlado por el Partido Nacionalista Vasco, no estuviera en deuda alguna con el PSOE es lo que posibilitó que los vascos pudieran impulsar su propia televisión, en enero de 1983. El vicepresidente del gobierno socialista Alfonso Guerra todavía decía lo siguiente unos meses después: 'En la agenda del gobierno no está la regulación de ninguna televisión independiente'. Pero el PSOE no pudo controlar los acontecimientos allá donde no disponía de mayoría. Las amenazas de Guerra eran inútiles en Euskadi" (Maxwell, 1995, 110). 
El teórico estadounidense asegura que el poder valerse del derecho a tener unos medios de comunicación propios tenía una gran importancia para los vascos, gallegos y catalanes para poder impulsar su cultura y su afianzar su "nacionalidad": "Para los vascos, gallegos y catalanes ello suponía un gran reto: revivir su nacionalidad para perdurar, seguir con vida. Su políticas culturales se impulsaban contra la cultura centralista del estado-nación". (Maxwell, 1995, 153).

El uso exclusivo de la lengua propia para difundir e impulsar la cultura y afianzar al mismo tiempo el hecho nacional puede suponer, en el caso vasco, una limitación, dado que casi tres cuartos de la población de la CAV no hablan la lengua vasca. Por esta razón ETB impulsó tres años después de su creación un canal en castellano, como recuerdan José Ignacio Armentia y José María Caminos en su libro La información. Redacción y estructuras:

"Con la intención de contribuir a la recuperación lingüística y cultural de Euskadi, Euskal Telebista nació como un único canal íntegramente en euskara. Sin embargo, la posterior necesidad de llegar a todos los ciudadanos vascos y abarcar mercados publicitarios más amplios, hizo que ETB pusiera en marcha, en mayo de 1986, un segundo canal con emisiones en castellano" (Armentia y Caminos, 1999, 75).

ETB2, el canal en español de la televisión vasca, fue creado por José María Gorordo cuando era director general del ente. Gorordo argumentó razones de audiencia y eficacia para defender la puesta en marcha del segundo canal. Sin embargo, Joan Mari Torrealdai declara que bajo esas justificaciones subyacían razones políticas: "Hace suyo (Gorordo) el discurso de aquellos que pedían que ETB emitiera también en castellano y propone que se superen las limitaciones lingüísticas, que el euskara no sea exclusivista y que ETB llegue al $100 \%$ de los ciudadanos que pagan sus impuestos. De ahí surge ETB2 y, según dicen las malas lenguas, de un deseo de poder tener una influencia política directa" (Torrealdai, 1993, 155).

Estos intentos de control político y del uso de la información mencionados por Torrealdai son una constante en la relación, siempre en mayor o menor medida tensa, entre los medios de comunicación y el poder. El control de la información es una fuente de poder. Esta constatación es más cierta, si cabe, en el ámbito de la información política, sobre todo en una sociedad tan politizada como la vasca. ETB no ha sido una excepción a esto, ya que tampoco ha estado libre de ese afán de controlar la gestión y la difusión de la información para así interpretar a su manera la realidad. Al fin y al cabo, las noticias y los informativos son una interpretación de la realidad, tal y como señalan Ramón Salaverría y Rafael Cores: "La labor interpretativa es consustancial al periodismo. Todo género periodístico supone, en mayor o menos medida, una interpretación de la realidad reflejada en un texto" (Salaverría y Cores, 2005, 165).

Este deseo de control queda en evidencia en el informe que presentó la dirección de ETB en el año 1992. El texto muestra los aspectos positivos del modelo televisivo que reivindican los gestores del ente público, pero también planteamientos menos loables, como asegura Ramón Zallo: comportamientos y actitudes criticables que nos pueden llevar a una modelo de información paragubernamental que nos acerca a un sistema informativo de tendencia más autoritaria:

"Es como para empezar a temblar cuando en las guías de los servicios informativos se leen términos o expresiones como 'tener en cuenta la repercusión social', 'informar basándose en nivel objetivo de implantación que, en general, tienen en la sociedad vasca' (cuando se refiere a las distintas fuerzas políticas) 'se evitará la ambigüedad informativa', 'se mostrarán los logros obtenidos por la actividad política y los aspectos positivos de esta como un medio adecuado para defender la democracia', 'hay que otorgar prioridad a lo institucional', 'no promover artificialmente la desconfianza ni el pesimismo', 'se promoverán el renombre y el prestigio de las instituciones' o "los servicios informativos hacen suya la política a favor de la paz del denominado Pacto de Ajuria-Enea”" (Zallo, 1993, 145).

Las críticas que Zallo realiza a la utilización política de la información en Euskal Telebista han sido una constante desde la creación del ente público. Este punto de vista también apunta a la política informativa impulsada por los socialistas en la televisión pública vasca a partir del año 2009. Fue entonces cuando el PSEEE llegó a la presidencia del gobierno vasco con la ayuda del Partido Popular y cambió los órganos de dirección de ETB: 
"El tema de la identidad: EI PSOE no tenía intención de tocarlo y mira: en vez de la ikurriña ha colocado la bandera española. Ha emitido el mensaje del rey de España en la ETB. Ha cambiado el mapa del tiempo de una manera absurda y patética. Dicen que la ETB tenía una identidad nacionalista, y que le ahora le han dado un toque de cosmopolitismo. Pues no. El toque que le han dado no es nada cosmopolita, sino español" (Iturriotz, 2010, 5).

Si bien hay matices e incluso diferencias importantes en algunos aspectos, no se puede afirmar que exista una división en términos totalmente opuestos a la hora de valorar ambos períodos. Basarse en una dicotomía blanco-negro sería fruto de la ingenuidad o, todavía peor, de la mala fe. No es pertinente asegurar que durante el mandato del PNV no había ningún tipo de manipulación política y que, sin embargo, con los socialistas se alcanzó la cima de la intoxicación informativa. Ni lo uno ni lo otro. La mayoría de los expertos en medios audiovisuales vascos -Amezaga, Torrealdai, Zallo- aseguran que en las dos épocas se ha producido el uso político de la televisión por parte del poder. Según estos críticos, en la época socialista esta tendencia se ha intensificado o, por lo menos, se ha hecho más evidente:

"Por lo que respecta a EITB hay que decir que el gobierno del PSE-EE ha encontrado un camino más fácil, más trillado, que en otros ámbitos. En el modelo que se ha construido durante 25 años, la televisión y la radio pública se han entendido como un instrumento en manos del partido que estaba en el poder, y entonces este gobierno ha hecho lo mismo que los anteriores: cambiar los puestos de responsabilidad y utilizar el medio para su propio beneficio. Esos casos de censura y vetos que tanto se mencionan ahora no los ha inventado este gobierno; se han dado con frecuencia en la historia de EITB. Es cierto que ahora esas tendencias se han hecho más evidentes, pero eso no debería hacernos olvidar todo lo anterior". (Amezaga, 2010, 16).

Han pasado ya treinta años desde que naciera la televisión vasca, y no se puede decir que el modelo criticado por Zallo haya cambiado sustancialmente: la información sigue siendo un instrumento para afianzar el modelo político, e incluso social, más fiel al ideario del partido que está en el gobierno aunque, más de acorde con los nuevos tiempos que se viven en el País Vasco -decisión de ETA de abandonar su "actividad armada" - esa política informativa puede haber variado en formas e intensidad.

\section{CUESTIONES DE LA INVESTIGACIÓN}

\section{Objetivos:}

Los objetivos de este estudio son tres: analizar la información política vasca en los informativos de la ETB durante el mandato del gobierno nacionalista y el socialista; comparar los resultados del análisis de los informativos de los años 2008 y 2010 para así apreciar las diferencias en los contenidos de ambos períodos y, por último, reflexionar e impulsar la discusión crítica sobre las políticas y criterios por los que se debería regir la información política en un medio de comunicación público.

\section{Hipótesis:}

Las hipótesis principales que este trabajo pretende demostrar son dos:

1) La información sobre política vasca difundida durante los períodos de gobierno de Juan José lbarretxe (PNV) y Patxi López (PSE-EE) como lehendakaris refleja visiones e identidades diferentes del País Vasco, enfrentadas entre sí: el primer concepto nos presenta un País Vasco acorde con una visión nacionalista, que incluye el territorio que, a ambos lados de los Pirineos y desde una perspectiva lingüística y cultural, se conoce como Euskal Herria. Por otro lado, tenemos el concepto constitucionalista español que identifica el País Vasco con el conjunto de los tres territorios integrados en la entidad denominada Comunidad Autónoma Vasca (CAV) o Euskadi.

2) Las características y la clasificación de los contenidos de los informativos varían en las dos épocas analizadas. En el período de Ibarretxe la politización del informativo es más evidente, no desde una perspectiva nacionalista vasca, sino desde el grado de importancia otorgado a la información política en general, mayor que el del periodo inmediatamente posterior. En el tiempo del gobierno socialista disminuyen el número de unidades de información políticas y la duración de estas. Eso produce una despolitización aparente de los contenidos del 
noticiero que, de hecho, podría también catalogarse como repolitización: traslada el foco de la atención informativa a un ámbito con unidades de información de contenido más social, con aumento de noticias curiosas y pintorescas para despojar así al Gaur Egun del mayor peso político que lo caracterizaba en la época anterior y, aparentemente, despolitizarlo.

\section{METODOLOGÍA}

Este trabajo ha analizado la edición de noche del informativo Gaur Egun, emitido en euskara, y se ha basado en los dos modelos de investigación habituales en este ámbito: el cualitativo y el cuantitativo.

\subsection{Investigación cualitativa}

Por medio del análisis cualitativo se han examinado las bases teóricas de la información política vasca difundida por ETB en el Gaur Egun. Para ello se han realizado dos focus groups y dos entrevistas en profundidad. Los focus groups ofrecen la oportunidad de obtener material contrastable y de gran valor, extraído de las conversación e interacción entre las personas que participan en ellos: "Definiremos entonces el grupo focal como una técnica de investigación social que privilegia el habla, cuyo propósito radica en propiciar la interacción mediante la conversación acerca de un tema u objeto de investigación, en un tiempo determinado, y cuyo interés consiste en captar la forma de pensar, sentir y vivir de los individuos que conforman el grupo" (ÁlvarezGayou, 2003, 138).

En esta investigación se han realizado dos grupos focales. En cada uno de ellos se han reunido seis personas: en el primero, los participantes pertenecían al mundo académico del ámbito de la comunicación audiovisual; en el segundo, se trataba de ciudadanos corrientes, telespectadores del informativo en cuestión, distribuidos por sexo y rango de edad. En ambos casos se ha guardado una distribución de los participantes por sexo. En el segundo focus group los voluntarios han sido elegidos, además de por representación paritaria por sexo y por su franja de edad, por la diversidad geográfica de su origen. El objetivo ha sido reunir un grupo de personas lo más heterogéneo posible.

Las entrevistas en profundidad son el segundo elemento utilizado en el análisis cualitativo para interpretar los datos obtenidos en la investigación. Estas entrevistas tienen un objetivo: lograr una imagen nítida del punto de vista que el entrevistado tiene sobre el tema de análisis. El entrevistado aparece como un experto y el entrevistador como un investigador cuyo objetivo es conseguir la mayor información posible de aquel. Andrea Fontana y James H. Frey señalan que el entrevistador no deberá influir en el discurso del entrevistado; no hará saber su opinión personal sobre el tema que se está tratando. Para ello, Fontana y Frey dan estos consejos al investigador: "Nunca hay que sugerir ninguna respuesta, ni asentir o disentir de alguna respuesta. No hay que proporcionar al entrevistado ninguna idea acerca del punto de vista personal que se tiene acerca del tema" (Fontana y Frey, 1994, 364).

Los protagonistas de estas dos entrevistas en profundidad han sido Jaime Otamendi y Roberto Montalvillo. Otamendi era director de los servicios informativos de ETB en el año 2008. Montalvillo ocupaba el mismo cargo en el año 2010, cuando la dirección del ente ya había cambiado después de que los socialistas llegaran al gobierno vasco.

\subsection{Investigación cuantitativa}

El análisis de contenido de este trabajo de investigación ha tenido como universo todos los ítems de los informativos Gaur Egun de la edición de noche de ETB emitidos desde el 1 de enero al 31 de diciembre del año 2008 y desde el uno de enero del 2010 al 31 de diciembre del mismo año.

En el año 2008 se emitieron 366 Gaur Egun en la mencionada edición -fue año bisiesto-, y 365 en el año 2010. La muestra seleccionada de ese universo se basa en el modelo de la semana compuesta de aplicación quincenal. En total se han tomado 24 informativos del 2008 y 25 del 2010, con un total de 1.210 unidades de información (UI). 552 UI corresponden al año 2008 y 710 UI, al 2010.

La unidad de información es la entidad utilizada como referencia para el análisis de contenido. Se trata de una 
pieza compuesta de elementos orales y visuales, en cualquier formato, emitida con o sin la introducción del presentador. Esta pieza está insertada en el corpus del informativo, entre la presentación con los titulares y la despedida, diferenciada del resto de unidades de información.

Mediante este procedimiento se ha creado una muestra que servirá para sustituir con fiabilidad el universo de los informativos en el análisis cuantitativo. "En la investigación científica, con frecuencia, se elige, mediante un procedimiento minucioso, una muestra representativa del universo que con anterioridad se ha definido con claridad. La palabra representativa cobra especial relevancia aquí, ya que el valor de la muestra reside en ser equivalente, sustituta del universo de la investigación" (Zabaleta, 1997, 154).

El eje de esta investigación es la información difundida sobre política vasca en el informativo Gaur Egun. Para entenderlo, es necesario explicar dos conceptos claves utilizados en el análisis, y que determinarán el estudio: política y País Vasco. Como política, el autor entiende aquella actividad de quienes rigen o aspiran a regir los asuntos públicos, y a la actividad del ciudadano cuando interviene en los asuntos públicos. En la sección de Política del informativo se incluirían las noticias relativas a esa actividad, tanto a nivel institucional como fuera de ese ámbito, y los conflictos inherentes a la gestión de esos asuntos públicos. Política vasca sería toda aquella actividad, realizada dentro o fuera del País Vasco, cuyo objetivo sea regir, aspirar a regir o influir en la gestión de los asuntos públicos de cualquier territorio del País Vasco.

Se entiende por País Vasco la totalidad de los territorios a ambos lados de los Pirineos en los que históricamente se ha hablado y en la actualidad se habla la lengua vasca: por un lado Álava, Bizkaia, Gipuzkoa y Navarra; por el otro el Labourd, la Baja Navarra y el País de Soule, integrados en el departamento francés de los Pirineos Atlánticos.

El contenido de los informativos se ha clasificado en seis secciones: política, sociedad, economía, internacional, cultura y curiosidades. Este último apartado lo constituyen las unidades de información que abordan temas pintorescos, anecdóticos, de gran impacto visual, cuya preponderancia es cada vez mayor en los informativos de la televisión. La sección de deportes se ha quedado fuera del área de investigación al diferenciarse del informativo valiéndose de otro formato o de un corte publicitario, según los casos.

Las informaciones han sido clasificadas en un sistema de doce categorías creadas al efecto por el autor de la investigación. Las categorías son las siguientes:

1) Noticia de apertura de informativo (si / no)

2) Noticia de los titulares del informativo (si / no)

3) Secciones

4) Mención a la territorialidad vasca en el informativo (País Vasco, País Vasco Sur, País Vasco Norte, Euskadi o Comunidad Autónoma Vasca, Navarra o Comunidad Foral Navarra, País Vasco Norte)

5) Protagonistas directos: representantes de los partidos o agentes políticos vascos que aparecen haciendo declaraciones en las unidades de información analizadas.

6) Protagonistas indirectos: representantes de los partidos o agentes políticos vascos que son mencionados en las declaraciones de los protagonistas directos en las unidades de información analizadas.

7) Foco territorial: origen geográfico de la unidad de información (UI) analizada.

8) Representación del País Vasco en las infografías y complementos icónicos del informativo.

9) Tipos de unidades de información: noticia o vídeo completo, noticia con aparición ante la cámara del periodista, noticia con conexión directa, noticia con presentador y voz en off, noticia con voz en off, corte con solo declaraciones.

10) Duración de las unidades de información: 0-45", 46"-90", 91"-120", +120". 
11) Lugar ocupado por la UI en la escaleta del informativo: 0-9, 10-18, 19-27, +27.

12) Distribución de la UI en el tiempo de duración del informativo: 0-10', 11'-20', 21'-30', +30'.

Para verificar la fiabilidad de este sistema se ha realizado una prueba piloto con tres personas que han mirado un informativo y han analizado y codificado sus unidades de información basándose en dicho sistema de categorías. Los resultados han avalado la utilidad del sistema, ya que los tres codificadores independientes han coincidido en un $96,89 \%$. "Los expertos en metodología afirman que entre los codificadores ha de haber como mínimo una coincidencia del 90\% para considerar idónea el sistema de categorías" (Zabaleta, 1997, 226).

\section{RESULTADOS}

Los resultados obtenidos al analizar los Gaur Egun de la muestra de los años 2008 y 2010 presentan diferencias evidentes entre los modelos de informativos de ambos. Estos resultados se aprecian en las tres grandes técnicas de investigación utilizadas en este trabajo: análisis de contenido, en el que se basa la parte cuantitativa del estudio, y en entrevistas en profundidad y focus group o grupos de discusión, fundamentales en la investigación cualitativa.

\subsection{Análisis de contenido:}

En el año 2008 predominaban las unidades de información o UI de índole política en general. Esta sección sumaba el $42 \%$ de todas las UI. El porcentaje baja hasta el $25 \%$ en el año 2010 . Estas diferencias son también perceptibles en las unidades de información localizadas geográficamente en el País Vasco. Por otra parte, en el año 2010 aumenta considerablemente el porcentaje de Ul clasificadas en la categoría de Curiosidades: En total suponen un $6 \%$ frente al $1 \%$ del año 2008 .

Unidades de información (UI) por secciones

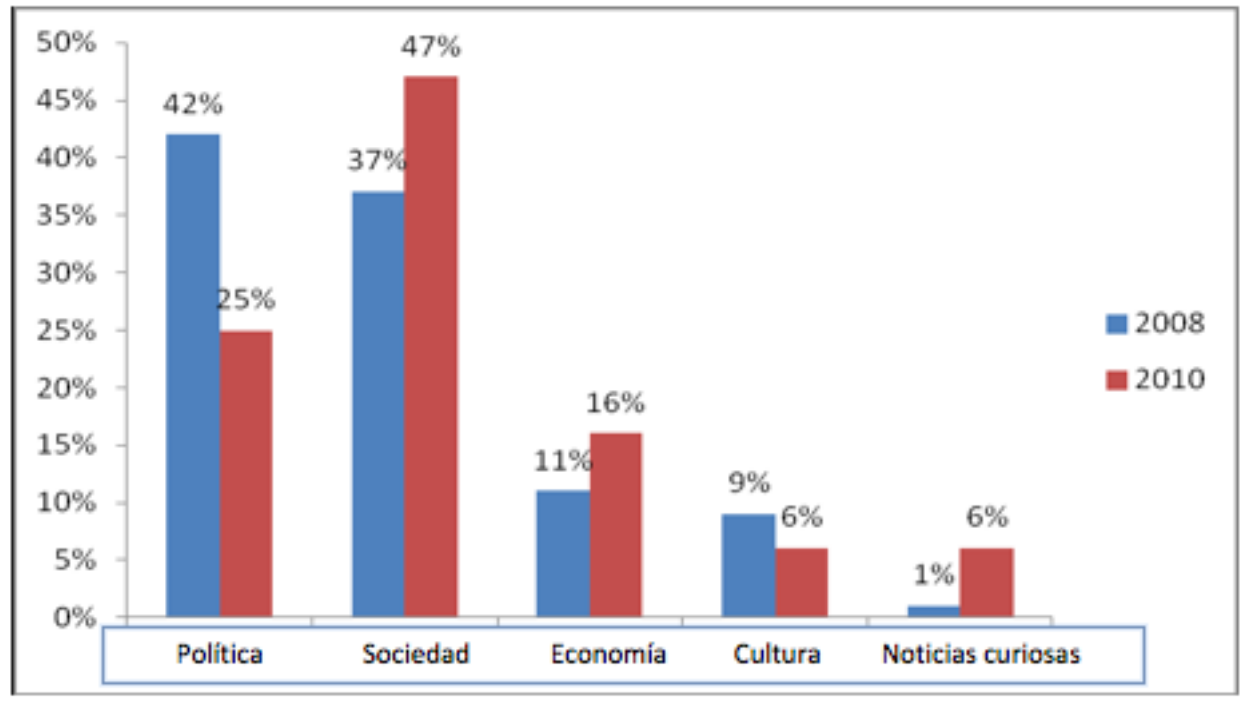

La Comunidad Autónoma Vasca ha sido el epicentro de la información política en ETB, tanto en la época de Ibarretxe como en la de López. Sin embargo, esta tendencia se acentúa en este segundo período, en el año 2010. Aumenta el porcentaje de las de las unidades de información cuyo origen se sitúa en la CAV. El porcentaje de aquellas que tratan sobre la actualidad política en la Comunidad Foral Navarra desciende a la mitad en ese mismo año si lo comparamos con el del año 2008. En ese sentido, se aprecia un deseo de restringir la información al ámbito territorial aceptado y denominado como vasco en la constitución española y el estatuto de autonomía de Gernika: las provincias de Álava, Bizkaia y Gipuzkoa.

En cuanto al formato o tipo de unidad de información utilizado en los informativos de las muestras analizadas, cabe destacar que en el año 2010 predomina el ítem denominado vídeo en off (47\%) frente al 34\% del año 2008. Las conexiones en directo a la hora de abordar temas de la política vasca descienden drásticamente en la época de López (4\%) comparadas con las efectuadas en la época anterior (20\%). El hecho de que el porcentaje de esas conexiones sea cinco veces mayor en el 2008 que en el 2010 denota que en el primer año 
se utilizan más medios técnicos y humanos y existe, en cierta medida, mayo interés en cubrir este tipo de información.

Tipos de UI en la información política vasca

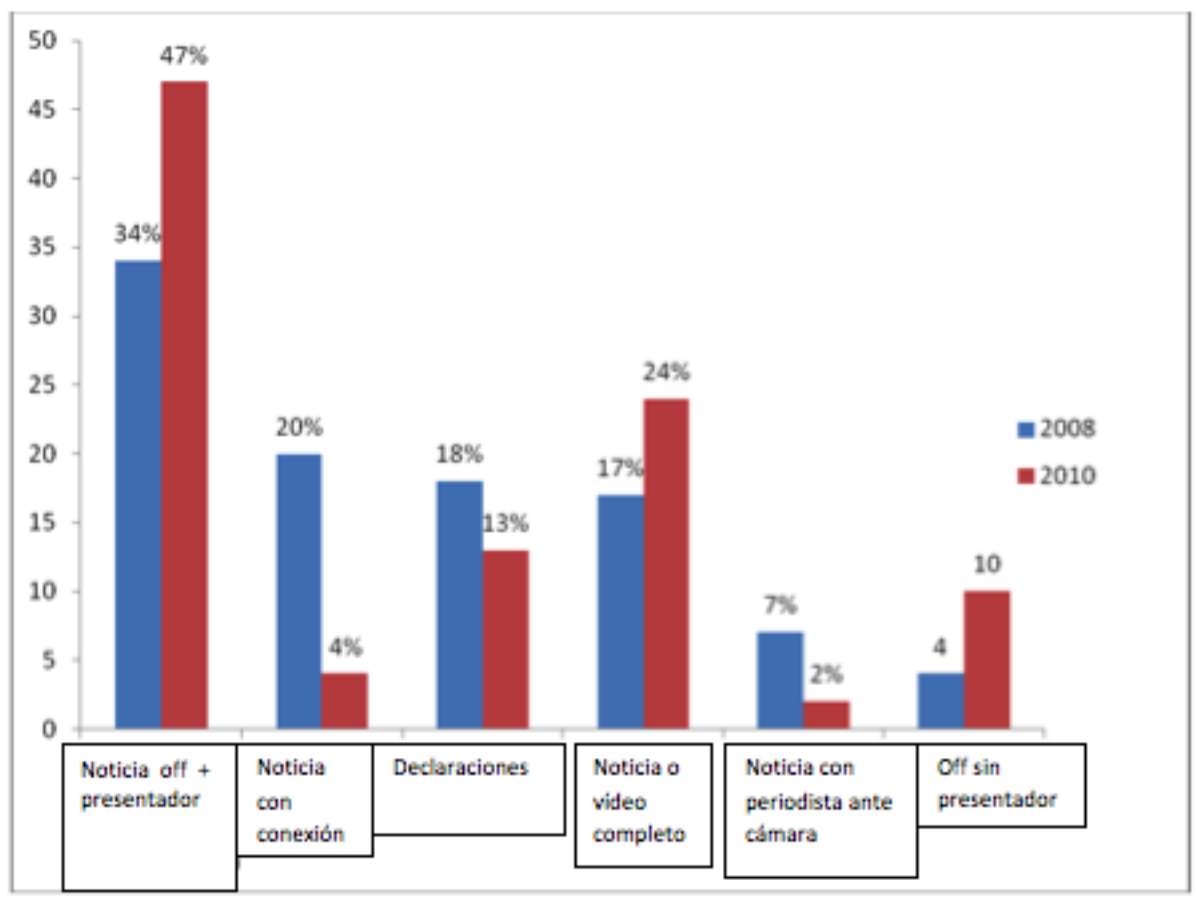

El análisis de la duración de las UI también nos aporta información sobre el nivel de importancia que se le da a la información política vasca. En el año 2010 casi tres cuartas partes de todos los ítems (72\%) tenían una duración de hasta 45 segundos como máximo. En cambio, en el 2008 las UI de este tipo no llegaban a la mitad (47\%). Las UI cuya duración oscila entre los 46 y 90 segundos son el $27 \%$ en el 2010 , diez puntos más que en el 2008. En el apartado de las UI con duración entre los 90 y 120 segundos solo tenemos un porcentaje del 1\% en el año 2010, pero el $13 \%$ en el 2008. Por último, en el año 2008 hay un porcentaje del 3\% de las UI de duración superior a los dos minutos. Este tipo de ítem desaparece en los informativos emitidos el año 2010.

Duración de las UI en la información política vasca

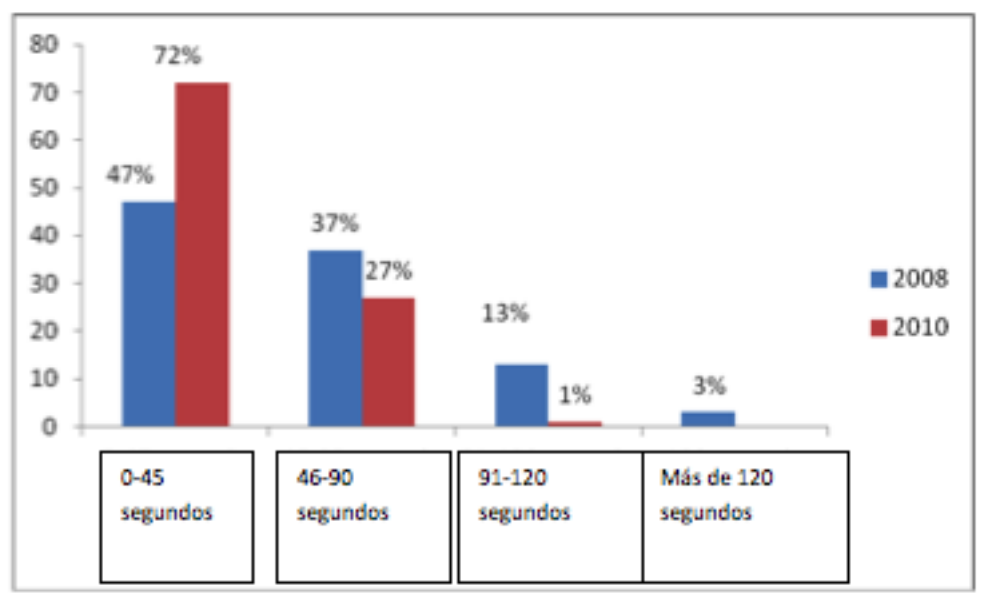

El análisis acerca de la distribución de las UI que tratan sobre la actualidad política vasca también muestra diferencias entre los dos años, y afianza la constatación de que el 2008 da mayor relevancia a este tipo de noticias. El orden de los ítems es más compacto y claro en el año correspondiente al mandato de Ibarretxe. La mayoría de los ítems, el 97\%, se encuentra entre las 18 primeras UI del informativo. Por el contrario, dos años después esas unidades de información aparecen distribuidas no solo al principio, sino también al final del informativo. En el 2010 se rompe la estructura tradicional del informativo y se salta de una sección a otra, alternando política, sociedad, economía e internacional. No hay compartimentos claros que reúnan las noticias correspondientes a cada sección, y esto hace que estas pierdan intensidad, fuerza, ya que sus contenidos se 
encuentran diseminados en el informativo, sin ocupar un lugar central, y diluidos a los ojos del telespectador, tal y como afirma Rosa Martín Sabaris en su tesis doctoral La organización informativa y los procesos de producción de la noticia. La información diaria en Euskal Telebista:

"Como norma general, puede decirse que la importancia decrece a medida que avanza el informativo, y que esto es así tanto para cada noticia aislada como para la disposición de las secciones dentro del programa. Se yuxtaponen así dos criterios para la colocación de las noticias en el guión: uno que clasificaría las secciones según su importancia (cultura es menos importante que política y, por consiguiente, se emite más tarde) y otro que ordenaría los temas dentro de la sección" (Martín Sabarís, 1999, 313).

La composición de los titulares también aporta información sobre la relevancia otorgada a la información política vasca en los informativos. En el año 2008 el 43\% de todos los titulares pertenecían a este apartado. La segunda sección más mencionada en los titulares era la de Sociedad (39\%); Estos porcentajes de invierten en el 2010. La política vasca pasa a ocupar un segundo puesto, con un $31 \%$, la inmensa mayoría de ellos pertenecientes a la CAV. En primer lugar se encuentran los titulares de Sociedad, que suponen un $49 \%$ del total.

El tema de la territorialidad y las diferencias que se aprecian entre los informativos que se han analizado del 2008 y del 2010 son dignos de mención y vienen a corroborar la idea de que existen dos conceptos diferentes del País Vasco en cada período. Por una parte, aquel que toma los territorios de ambos lados de los Pirineos como unidad y los engloba como un ente común, unidos por vínculos lingüísticos, culturales e históricos. Para ese sector, cuya filosofía se refleja claramente en la muestra de los Gaur Egun del 2008, el País Vasco, Euskal Herria, lo componen Álava, Bizkaia, Gipuzkoa, Navarra, Lapurdi, la Baja Navarra y Zuberoa. Por otro lado, el que considera que, ateniéndose al orden constitucional, el País Vasco incluye solo las tres provincias de la CAV.

Estos dos conceptos tan diferentes se ven reflejados en los informativos analizados. En el año 2008 el concepto Euskal Herria es el más presente en las referencias geográficas orales de los periodistas. El concepto de Comunidad Autónoma Vasca o Euskadi como equivalente a este apenas es utilizado. En los informativos del 2010 ocurre todo lo contrario: la inmensa mayoría de las referencias, el $86 \%$, se refiere a la CAV; el $8 \%$ a Euskal Herria y el 6\% a Ipar Euskal Herria, literalmente País Vasco Norte, allende los Pirineos. Hay que señalar que cuando en el año 2010 se menciona Euskal Herria normalmente se hace en un sentido estrictamente cultural, con noticias relacionadas a la situación del euskara y asuntos relacionados con esta, a la literatura escrita en esta lengua, costumbres etc. Nunca se le otorga valor político alguno a este concepto. En el 2010 también desaparece del informativo el concepto Hego Euskal Herria, País Vasco Sur, que engloba a la CAV y a Navarra. En el 2008 esa denominación se encontraba en un 13\% de las referencias orales a la territorialidad.

En las infografías sucede lo mismo. Las del año 2010 se ciñen rigurosamente al modelo constitucional vigente en el estado español y nos presentan la división por comunidades autónomas. La Comunidad Autónoma Vasca y la Comunidad Foral Navarra aparecen separadas en todas las infografías de ese año. En los informativos del 2008 la tendencia es la contraria: en el $72 \%$ de los casos aparecen las siete provincias sin ninguna señal que delimite sus fronteras administrativas; en un $14 \%$ de la infografías aparece Hego Euskal Herria unificada, con Álava, Bizkaia, Gipuzkoa y Navarra, sin ninguna separación administrativa, todo lo contrario de lo que sucede en el $14 \%$ de las infografías restantes.

Mención especial requiere el mapa del tiempo. En el año 2008 el mapa del tiempo de Euskal Telebista incluye únicamente las provincias de Álava, Bizkaia, Gipuzkoa, Navarra, Lapurdi, Baja Navarra y Zuberoa, sin ningún tipo de referencia a España o Francia. El mapa que dos años más tarde nos señala las previsiones meteorológicas es totalmente distinto. Sigue conteniendo estos siete territorios, pero no como una unidad, ya que junto a ellos aparecen también España y Francia: la comunidad autónoma de la Rioja y las Landas. No es una decisión neutra, como tampoco lo fue la que en los albores de la ETB compuso el mapa del tiempo exclusivamente con los siete territorios antes mencionados.

"Los estados, y también los pueblos sin estado, se percataron hace mucho tiempo del valor estratégico de los mapas. El reino o estado que se apoderaba de un territorio y el pueblo que, pese a carecer de poder, quería construir un estado propio ya sabían el valor añadido que los mapas aportan (...) En las escuelas y en los 
medios de comunicación la imagen del mapa es un exponente claro del sentimiento de identidad nacional" (Basterretxea, 2000, 195).

Mapa del tiempo en el informativo del año 2008

Mapa del tiempo en el informativo del año 2010

\section{2: Entrevistas en profundidad:}

Las declaraciones realizadas por las dos personas protagonistas de las entrevistas en profundidad muestran las diferencias de criterio y enfoque informativos en el noticiero de los dos años estudiados. He aquí las opiniones de los directores de informativos de la televisión vasca acerca de los diversos aspectos analizados. Estas citas se clasifican en tres apartados: representación y control político; estructura y contenido del informativo y, por último, imaginario representado en el informativo.

\subsection{1: Representación y control político}

Jaime Otamendi (director de informativos de ETB en el año 2008):

"No sé con exactitud cuál es la tendencia actual, las apariciones de los responsables políticos; siempre hay que andar con cuidado con los datos. Yo sí que tengo la impresión de que las apariciones de la izquierda abertzale han descendido mucho, pero de los demás partidos, no sé, de verdad".

“¿Que el PP aparece mucho en los informativos? Bueno, eso es la impresión que se tiene desde fuera, pero si, seguramente será así. Ahí hay un acuerdo de gobierno, y el PP presionará lo suyo, seguro. Cuando se cambió la dirección de esta casa el PSOE colocó a su gente $t$ el PP

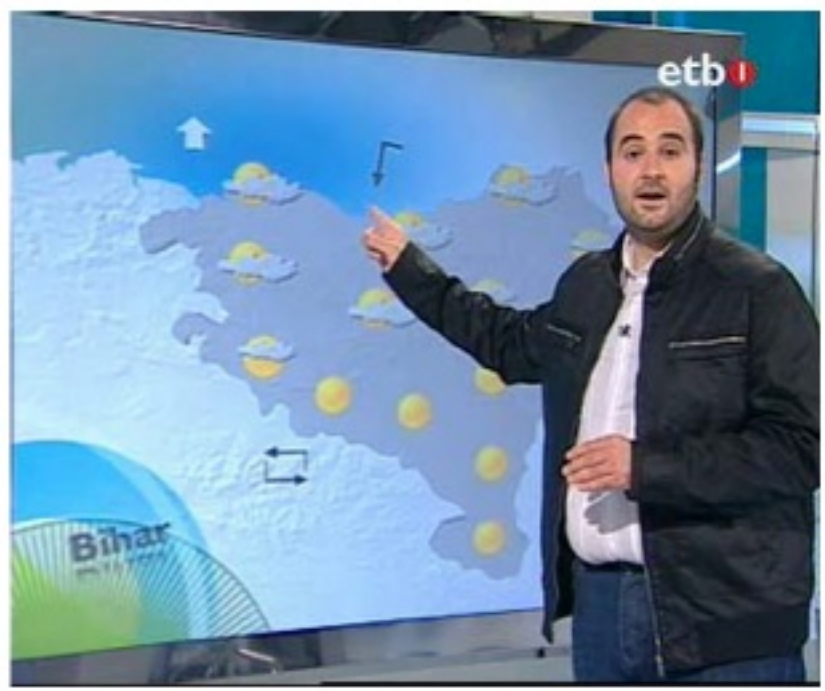
a la suya, y eso se nota".

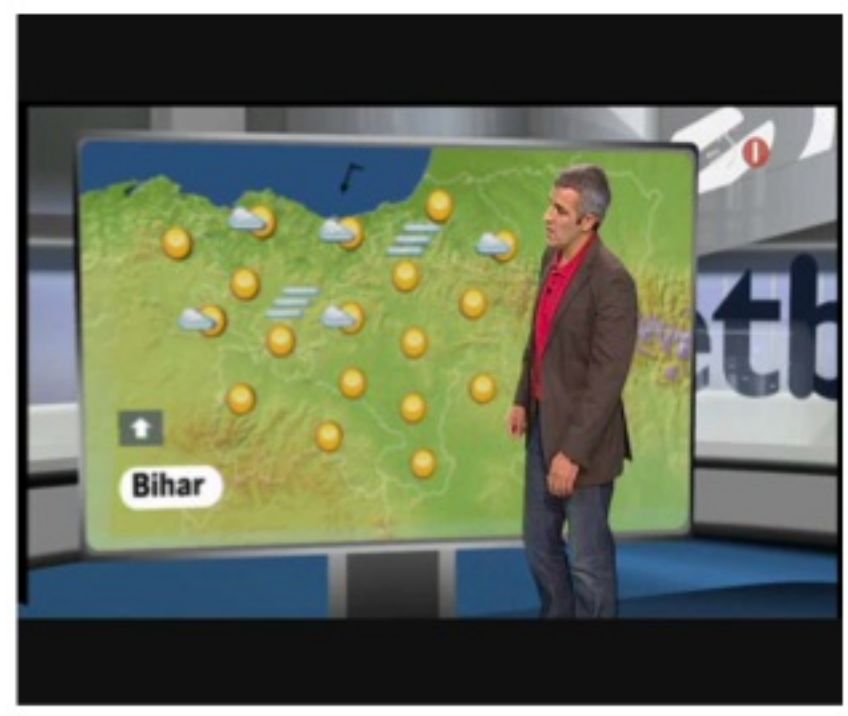

"En nuestra época en dirección pretendimos reducir la información política, pero aquí siempre ha habido muchas luchas entre los partidos políticos. Entonces, al final, cada día siempre había algún tema, una excusa para hacer una ronda y recabar la opinión de los partidos políticos".

Roberto Montalvillo (director de informativos de ETB en el año 2010):

"Nosotros no le damos menor importancia a la información política, pero tampoco estamos obligados a difundirla siempre en los primeros lugares del informativo".

"Es cierto que para reflejar los equilibrios políticos tienes que guiarte por la representación parlamentaria de esos grupos políticos".

“Los informativos reflejan la legalidad, y la izquierda abertzale es ilegal, no está representada en el parlamento".

\subsection{2: Estructura y contenido del informativo}

Jaime Otamendi (director de informativos de ETB en el año 2008):

"La televisión depende de las audiencias, es esclava de ellas. En algunos momentos, cuando ves que tienes un 
problema de audiencia, empiezas a tensar la estructura del informativo de otra manera. Dentro de esa dinámica puede suceder que cometas algunas locuras, eso pasa, si".

"Tener gran nivel de audiencia es importante, pero esa dependencia crea también mucha ansiedad (...) Si no tienes bien claros tus objetivos, y no tienes un poco de paciencia, y en los medios de comunicación no abunda la paciencia, pues haces tonterías para conseguir audiencia. Yo viví una época difícil de esas, y entonces crees que con noticias de sucesos e imágenes morbosas vas a solucionar el problema. Eso puede dar resultados, pero esos resultados solo son a corto plazo".

Roberto Montalvillo (director de informativos de ETB en el año 2010):

"Cambiamos los presentadores; también cambiamos los grafismos y los hicimos más modernos. Nos esforzamos en trabajar mucho la portada. Reunimos la información en bloques y entre esos bloques insertamos otras informaciones para aligerar el informativo".

“Jugamos mucho con la imagen. Esa tendencia a incluir en el informativo noticias curiosas, llamativas puede venir por distintas razones. Puede influir el gusto del editor, porque quizás piensa que se necesitan algunos momentos de relajación para después continuar con la narración, de una manera más adecuada. O quizás piensa que hay que terminar el informativo de otro modo, no de una manera brusca".

\subsection{3: Imaginario representado en el informativo}

Jaime Otamendi (director de informativos de ETB en el año 2008):

"Nosotros queríamos informar tomando el País Vasco en su totalidad: El País Vasco del Norte, Navarra y la Comunidad Autónoma Vasca. Intentamos hacer un informativo global, es decir, que no estuviera compuesto por noticias locales. Queríamos ofrecer una visión general, para que el espectador no tuviera la necesidad de ver otro informativo".

"En aquella época se podía decir Euskal Herria, englobando el País Vasco en su totalidad, más en el informativo en euskara que en el emitido en castellano. En vasco no había ningún problema. En el informativo en castellano a veces se utilizaba Euskadi para llamar a la Comunidad Autónoma Vasca. Euskadi y Navarra. Nosotros intentábamos agrupar los datos de todo el País Vasco Sur, la CAV y Navarra, pero eso no era fácil".

Roberto Montalvillo (director de informativos de ETB en el año 2010):

"Si nos basamos en la lógica, tú no puedes decir Euskal Herria (País Vasco con siete territorios culturales e históricos) si sólo estás dando los datos de Euskadi (Comunidad Autónoma Vasca). Eso es incorrecto. Nosotros nos ceñimos a la legalidad, Y si nos ceñimos a la legalidad, si estamos estructurando por un lado Euskadi y por otro Navarra, no es lógico hablar de Euskal Herria. No hay información política que abarque los siete territorios. Si la hubiera... pero no la hay".

"Nosotros nos basamos en la legalidad. Si el concepto es Comunidad Autónoma Vasca, nosotros nos basaremos en la Comunidad Autónoma Vasca".

"Todavía seguimos haciendo referencia a Navarra, pero sí que es cierto que situamos el foco de atención en ese orden: primero, Euskadi; luego, Navarra”.

\section{3: Grupos de discusión}

Las opiniones de las personas presentes en los focus group sirven para avalar las hipótesis del estudio. Los puntos de vista de los participantes en estos grupos de discusión se ofrecen englobados en los tres mismos apartados utilizados para resumir las entrevistas en profundidad.

\subsection{1: Representación y control político}

"Antes el contenido era más abertzale; ahora hay más información española. La información de aquí no ocupa tanto espacio como antes" (Agurne Agirregomezkorta, participante del segundo grupo de discusión). 
"Los informativos del 2010 tienen un contenido más ligero, incluso superficial. No hay nada que se pueda sujetar con fundamento, algo en lo que se profundice. Es cierto que una sola noticia no sirve para profundizar en un tema, que a duras penas se puede llegar a contextualizar el hecho narrado, pero ahora cada noticia vapor libre. Ahora no sé cuál es el papel del editor, si ha cambiado, o si han cambiado a los editores y no les han dado el rol que tenían antes" (Beatriz Zabalondo, participante del primer grupo de discusión).

"Me parece que ahora, en la época de López, los periodistas tienen más miedo a tratar noticias políticas. Igual no han cambiado mucho, pero en la época del PNV se trataban unos temas determinados y los periodistas no tenían problemas para informar de noticias de temática abertzale" (Joseba Erremundegi, participante del segundo grupo de discusión).

"En el informativo se instala un formato determinado en el que el editor controla muy bien qué corte, qué declaraciones del político utiliza, y entonces el papel del periodista es mecánico. 'De este segundo a este segundo', se le dice para que incluya el corte con las declaraciones, porque igual el responsable de un partido político ha llamado o porque ha dicho: 'hay que meter esto'. Y entonces, el papel del periodista es cada vez más débil. Al final el control del editor sobre el contenido político del informativo es una especie de censura" (Miren Gabantxo, participante del primer grupo de discusión).

"El modo de trabajar en la televisión vasca se basa en el trabajo de los comisarios políticos. La primera línea de la dirección de EITB (Radio-Televisión Vasca) en el año 2012 es del Partido Socialista, y la segunda línea, la ejecutiva, es del PP. Es decir, los unos controlan a los otros, Unos mandan más que otros, pero no pueden mandar sin saber que están siendo controlados por los otros. Por consiguiente hay una gran confusión a la hora de decidir qué es importante y qué no. Hay un problema grave de falta de coherencia: un profesional que es de izquierdas, o supuestamente de izquierdas, está bajo la supervisión o bajo el control de un profesional de derechas" (Ramon Zallo, participante del primer grupo de discusión).

"Antes dominaba el PNV, está claro. Después, el PSOE y el PP, y eso hace aparecer al PNV a un nivel más modesto, más pequeño. Parece que ha habido un cambio tremendo, pero igual no ha sido así. Por otro lado, yo soy navarro y veo que la mayoría de las veces salen los miembros del Parlamento Vasco, y parece que la información política de Navarra o de los otros territorios vascos no tenga importancia, los políticos de allí apenas aparecen. Bueno, a veces sale Yolanda Barcina, pero los otros, no" (Iker Tubia, participante del segundo grupo de discusión).

“El asunto de la representación política es un problema estructural desde el nacimiento de ETB. Tienen una comisión para mantener el equilibrio, pero no funciona como debiera. En Inglaterra sí que tienen fama de ser bastante imparciales. La televisión vasca debería haber tomado como modelo es ese aspecto algún modelo de fuera, pero tomaron el de la televisión española. Es muy triste. El control político que tenemos en nuestra televisión es parecido al que existe en la televisión española, bananero. Si vas a África, verás al dictador de turno todo el día saliendo en la televisión. Aquí no es muy diferente. No hay control para frenar eso. No hay alternativa. Algunos critican esta tendencia, pero cuando llegan al poder, ellos hacen lo mismo" (Patxi Alaña, participante del segundo grupo de discusión)

\subsection{2: Estructura y contenido del informativo:}

"Antes estaba más claro qué era noticia y qué no. Ahora parece que cualquier cosa es válida en el informativo. Además, no existe un orden lógico: si quieres introducir algún hecho curioso en el informativo, pues lo dejas para el final, bien diferenciado, y ya está. Pero al ponerlo todo en ese caos, pues todo queda como perdido, y el telespectador también se pierde con esos contenidos" (Estitxu Garai, participante del primer grupo de discusión).

“En general se aprecia eso, noticias sin fuste, sin razón de ser. Luego, la organización, la estructura del informativo, tampoco es clara. Antes algunos temas estaban mejor organizados: los de aquí, los de internacional... Ahora, no". (Patxi Alaña, participante del segundo grupo de discusión).

"Ahora, con el PSOE y PP, han cambiado el foco y las prioridades de la información, y eso crea confusión. Antes, te sentabas para mirar el informativo y ya sabías que las noticias más importantes aparecían en los 
primeros diez minutos. Ahí estaba lo más importante del día. Ahora no lo sabes. Ahora, igual ves el informativo completo y no hay nada que merezca la pena". (Beatriz Zabalondo, participante del primer grupo de discusión).

"La estructura y los contenidos se repiten. Las noticias del informativo del mediodía se vuelven a incluir en el de la noche, sin cambios. Si sucede algo especial, si hacen algo, pero tampoco se esfuerzan por explicar a la audiencia qué está sucediendo" (Pakita Lizarribar, participante del segundo grupo de discusión)

\subsection{3: Imaginario representado en el informativo}

"Creo que en el informativo de la época de López han jugado a difundir información digamos 'neutral'y, por otro lado, información dosificada. Para mí, lo más grave ha sido el referente de opinión pública que han utilizado, porque, en mi opinión, no es el referente de la opinión pública de aquí" (Ramon Zallo, participante dek primer grupo de discusión).

"Yo también he percibido algo así como una época de transición. Otra cosa es cuál es mi opinión al respecto. A mí me parece que el imaginario del País Vasco que se difundía en los informativos de la época de lbarretxe también era artificial. Pero sí, sí que he notado un cambio" (Patxi Doblas, participante del segundo grupo de discusión).

"Ahora en primer lugar sacan las tres provincias de la CAV y luego, aparte, Navarra. Después viene Iparralde, pero en otro nivel. En mi opinión las noticias de España las dan antes que las de Iparralde o País Vasco Norte" (Nahiara Mugertza, participante del segundo grupo de discusión).

"Antes también aparecían divididos el sur y el norte del País Vasco. Ahora eso es todavía más normal, sobre todo la división administrativa que hay en el sur entre Navarra y las otras tres provincias. Al difundir la información, primero tenemos Álava, Bizkaia y Gipuzkoa, y luego Navarra, incluso en las cosas más simples. Que está nevando... pues primero las tres provincias, detrás va Navarra y luego, España. Antes ya existía esta división, pero ahora está aún más clara" (Iker Tubia, participante del segundo grupo de discusión).0

\section{CONCLUSIONES}

Como se ha señalado en el apartado de la metodología, las hipótesis de este trabajo son dos:

1) Que la información sobre política vasca difundida durante los períodos de gobierno de Juan José lbarretxe (PNV) y Patxi López (PSE-EE) como lehendakaris refleja visiones e identidades diferentes del País Vasco, enfrentadas entre sí.

2) Que las características y la clasificación de los contenidos de los informativos varían en las dos épocas analizadas. En el período de Ibarretxe la politización del informativo es más evidente; en el tiempo del gobierno socialista disminuyen el número de unidades de información políticas y la duración de estas, lo que produce una impresión de despolitización.

Vistos los datos del estudio, las conclusiones extraídas vienen a corroborar las dos hipótesis de este trabajo, arriba mencionadas:

- Los contenidos y el enfoque político de los informativos Gaur Egun en el periodo de Ibarretxe y durante el mandato de López difieren sustancialmente, ya que el primero pretende reflejar el País Vasco desde el imaginario político nacionalista, abarcando los siete territorios a ambos lados de los Pirineos; el segundo aboga por defender la españolidad de ese territorio, acotando la información y circunscribiéndola al ámbito geográfico de la Comunidad Autónoma Vasca o Euskadi.

- En comparación con los informativos del año 2008 (época lbarretxe), en los Gaur Egun del 2010 (época López) la información política pierde protagonismo en favor de otras secciones, como puede ser la correspondiente a la información de sociedad. La información política vasca ya no abre necesariamente el informativo, como venía siendo habitual en el período anterior. El hecho de que esta no ocupe los primeros lugares del informativo, como era normal en la época anterior conlleva una inversión del orden en el que las secciones del informativo se sucedían en el pasado, lo que puede llevar a pensar al espectador que ese tipo de 
información ha pasado a un segundo plano. Al fin y al cabo, las unidades de información aparecen siempre concatenadas una detrás de otra siguiendo la lógica imperante interiorizada por el espectador, según la cual las noticias de mayor importancia se difunden al inicio del informativo o en los primeros minutos de este. Por otra parte, la duración de las unidades de información que abordan noticias políticas del País Vasco, tipos de ítems utilizados en ellas, y la ubicación de estos en la estructura del informativo también nos muestran diferencias en el año 2010 respecto al 2008: la duración de las unidades de información de política es menor en el 2010; predominan los vídeos en off y estas noticias no se disponen en un bloque claramente definido, sino que se suceden a lo largo del informativo, en una especie de efecto Guadiana.

- En el año 2010 la información sobre la actualidad política vasca aparece más restringida por lo que respecta a su origen geográfico. El modelo territorial de articulación autonómica inspirado en la constitución española se impone en los contenidos de los informativos del año 2010 a la hora de tratar sobre la actualidad política vasca. Esa legalidad se inscribe dentro del marco de la constitución española y el estado de las autonomías. Por ello, la información política vasca se considera como aquella originada en la CAV, es decir, en Álava, Bizkaia y Gipuzkoa.

- Las características de los arquetipos de los informativos de los dos años analizados muestran las diferencias entre ambos. El Gaur Egun del 2008 tiene una voluntad de reflejar el País Vasco desde una perspectiva nacionalista vasca. Pese a ceñirse a la legalidad, construye el imaginario nacional vasco a partir de la afirmación simbólica de los siete territorios que, según esta perspectiva, constituyen Euskal Herria desde un punto de vista lingüístico-cultural y que se proyecta también en el ámbito político. El informativo del 2010 refleja otra tendencia. Se mueve en la más estricta legalidad constitucionalista para insertar el País Vasco, delimitado a las tres provincias de la CAV, dentro de la realidad institucional y política española. Para ello, distingue claramente la Comunidad Autónoma Vasca de la Comunidad Foral Navarra y diluye así el carácter abertzale inherente al modelo del 2008.

\section{BIBLIOGRAFÍA}

Álvarez-Gayou Jurgenson, J. L. (2003). Cómo hacer investigación cualitativa. Fundamentos y metodología. Madrid: Paidós.

Amezaga,J. (2010). “Urtebeteko fikzioa”.Lasarte-Oria: Larrun n. 144, p. 15-16.

Armentia, J. I.; Caminos, J. M. (1998). La información. Redacción y estructuras. Bilbao: Universidad del País Vasco, Servicio de Publicaciones.

Basterretxea, J. I. (2000). "Lurraldetasuna: Euskal Herriaren forma-aniztasun mediatikoa” En: Nor Ikerketa Taldea. Hedabideak eta Euskal Herria. Bilbao: UEU, p. 155-197.

Ferrés J. (1994). Televisión y educación. Barcelona: Paidós.

Fontana, A.; Frey, J. (1994). "Interviewing: The Art of Science" En Denzin, N.; Lincoln, Y., The Handbook of Qualitative Research. Thousand Oaks, California, EEUU: Sage Publications, p. 361-376.

Iturriotz, A. (2010). “Normalizazioaren esperoan. PSEren eta PPren Ituna transbertsalitate bakarra”.Lasarte-Oria: Larrun n. 144, p. 2-14.

Martín Sabarís, R. M. (1999). La organización informativa y los procesos de producción de la noticia. La información diaria en Euskal Telebista. Bilbao: Universidad del País Vasco, Servicio de Publicaciones.

Maxwell,R. (1995). The Spectacle of Democracy. Spanish Television, Nationalism and Political Transition. Minneapolis, EEUU: University of Minnesota Press.

Salaverría, R., Cores, R. (2005). "Géneros periodísticos en los cibermedios hispanos" En: Salaverría, R. (coord.) Cibermedios. El impacto de internet en los medios de comunicación en España. Sevilla: Comunicación Social ediciones y publicaciones, p. 146-185. 
Torrealdai,J. M., (1993). “ETB eta euskara. Hamar urteren bueltan”. Jakin. 75, p. 151-159.

Zabaleta, I. (1997). Komunikazioaren ikerkuntzarako metodologia, Bilbo: UEU.

Zallo, R.(1993). “ETB: Txosten bati buruzko txostena”. San Sebastián: Jakin, 89, p. 135-150.

\section{Breve semblanza del autor}

José Mari Pastor González (Barakaldo,1961) es profesor del Departamento de Periodismo de la Universidad del País Vasco y doctor en Ciencias Sociales y de la Comunicación por la misma universidad. Ha trabajado durante más de 30 años en medios de comunicación tanto en lengua vasca como en castellano. Galardonado con el Premio de Periodismo Rikardo Arregi, en la actualidad compagina la docencia universitaria con su labor de columnista en prensa vasca y de colaborador de medios de comunicación como la ETB, Televisión Vasca. Participa en proyectos de investigación reconocidos y financiados por la Universidad del País Vasco.

Ámbitos. Revista Internacional de Comunicación, n.26, año 2014, tercer trimestre (otoño).

Recibido: $12 / 6 / 2014$

Aprobado: 4/7/2014 